\title{
The Involvement of RhoA and Wnt-5a in the Tumorigenesis and Progression of Ovarian Epithelial Carcinoma
}

\section{Shuo Chen ${ }^{1}$, Jun Wang ${ }^{2}$, Wen-Feng Gou ${ }^{3}$, Ying-Ling Xiu ${ }^{1}$, Hua-Chuan Zheng ${ }^{3}$,} Zhi-Hong Zong ${ }^{3}$, Yasuo Takano ${ }^{4}$ and Yang Zhao ${ }^{1{ }^{*} *}$

1 Department of Gynecology, the First Affiliated Hospital of China Medical University, Shenyang 110001, China; E-Mails: chenshuo077003@163.com (S.C.); xiu561480485@163.com (Y.-L.X.)

2 Department of Gynecology, the General Hospital of Shenyang Military Region, Shenyang 110045, China; E-Mail: wj202fck@163.com

3 Department of Biochemistry and Molecular Biology, Institute of Pathology and Pathophysiology, College of Basic Medicine, China Medical University, Shenyang 110001, China; E-Mails: xiaogouaeiou@163.com (W.-F.G.); zheng_huachuan@hotmail.com (H.-C.Z.); zzh660526@sina.com (Z.-H.Z.)

4 Clinical Cancer Institute, Kanagawa Cancer Center, Yokohama 241-0815, Japan; E-Mail: ytakano@gancen.asahi.yokohama.jp

* Author to whom correspondence should be addressed; E-Mail: yida.zhaoyang@163.com; Tel.: +86-139-4014-0056.

Received: 29 August 2013; in revised form: 26 November 2013 / Accepted: 3 December 2013/ Published: 12 December 2013

\begin{abstract}
Background: Ras homolog gene family member $\mathrm{A}(\mathrm{RhoA})$ is involved in Wnt-5a-induced migration of gastric and breast cancer cells. We investigated the roles of RhoA and Wht-5a in ovarian carcinoma. Methods: RhoA and Wnt-5a mRNA and protein expression in normal fallopian tube epithelium, benign tumors, primary ovarian carcinomas, and metastatic omentum were quantified. RhoA or Wnt-5a was knocked down in OVCAR3 ovarian carcinoma cells using siRNAs and cell phenotype and expression of relevant molecules were assayed. Results: RhoA and Wnt-5a mRNA and protein expression were found to be significantly higher in metastatic omentum than in ovarian carcinomas, benign tumors, and normal fallopian tube epithelium $(p<0.05)$, and positively associated with differentiation and FIGO staging (stage I/II vs. stage III/IV) in ovarian carcinoma $(p<0.05)$. RhoA and $W n t-5 a$ expression were positively correlated in ovarian carcinoma $\left(p=0.001, R^{2}=0.1669\right)$. RhoA or Wnt-5a knockdown downregulated RhoA and
\end{abstract}


Wnt-5a expression; reduced cell proliferation; promoted $\mathrm{G}_{1}$ arrest and apoptosis; suppressed lamellipodia formation, cell migration, and invasion; and reduced PI3K, Akt, p70S6k, Bcl-xL, survivin, and VEGF mRNA or protein expression. Conclusions: This is the first demonstration that RhoA and $W n t-5 a$ are associated with ovarian carcinogenesis and apoptosis inhibition; there might be positive correlation between RhoA and Wnt-5a expression. RhoA is a potential tumorigenesis, differentiation, and progression biomarker in ovarian carcinoma.

Keywords: ovarian carcinoma; RhoA; Wnt-5a signaling; tumorigenesis and progression; phenotype

\section{Introduction}

Ovarian cancer is a malignant tumor that represents a serious threat to women's health and is one of the leading causes of cancer-related deaths in females. More than $90 \%$ of ovarian cancers are classified as epithelial and are believed to arise from the ovarian epithelium [1]; however, evidence suggests that the fallopian tube inner surface epithelium may also be the origin of some ovarian cancers [2]. The risk factors for ovarian cancer include family history of ovarian carcinoma and genetic mutations. Ovarian cancer has a disproportionately poor survival rate due to the late onset of symptoms. Increased understanding of the relevant molecular mechanisms that regulate ovarian carcinogenesis may result in improved methods of diagnosis, treatment, and prevention.

Ras homolog gene family member A $(R h o A)$ is a small $(\sim 22 \mathrm{kDa}) \mathrm{G}$ protein/guanosine triphosphatase that is part of the Ras-related C3 botulinum toxin substrate (Rac) subfamily of the Rho family [3]. RhoA can reorganize the cell cytoskeleton and regulate cell migration by activating effector proteins such as Rho-associated coiled-coil kinase (ROCK) [4]; such changes are associated with tumor invasion and migration in several types of carcinoma cells [5,6]. Phosphoinositide 3-kinase/protein kinase B (PI3K/Akt)-dependent phosphorylation of glycogen synthase kinase-3 $\beta$ (GSK3 $\beta$ ) and RhoA activation regulate Wnt-5a-induced gastric cancer cell migration [7], in line with results for breast cancer cells [8]. Wnt-5a is a representative ligand for the seven-transmembrane receptor frizzled-5 and the tyrosine kinase orphan receptor 2 , activating $\beta$-catenin-independent pathways and regulating a variety of cellular functions, such as proliferation, differentiation, migration, adhesion, and polarity [9]. Marked expression of Wnt-5a has previously been reported in human metastatic melanoma and a variety of human primary tumor samples. However, the exact nature of its role in ovarian carcinoma remains undetermined.

To clarify the role of RhoA and Wnt-5a in ovarian carcinogenesis and subsequent progression, we examined their mRNA and protein expression in normal fallopian tube epithelium, benign tumors, primary ovarian carcinomas, and metastatic ovarian carcinomas simultaneously, the first time such an investigation has been carried out, and compared them with the clinicopathological features of patients with ovarian carcinoma. To study the molecular mechanisms, we observed the effects of RhoA or Wnt-5a knockdown on the phenotypes and expression of molecules regulated in ovarian carcinoma cells in vitro. 


\section{Results}

\subsection{Correlation of RhoA and Wnt-5a mRNA and Protein Expression with Pathogenesis and Aggressiveness of Ovarian Carcinoma}

RhoA and Wnt-5a mRNA and protein expression were quantified in normal fallopian tube epithelium, benign cystadenoma (serous and mucinous), primary ovarian carcinomas, and metastatic omentum using real-time polymerase chain reaction (PCR) (Figure 1A,B) and western blotting (Figure 1I). RhoA mRNA and protein expression were significantly higher in ovarian carcinomas than in benign tumors or normal fallopian tube epithelium (Figure 1C,J, $p<0.05$ ) and in metastatic omentum foci than in ovarian carcinomas (Figure 1D,K, $p<0.05$ ); similar trends were observed for Wnt-5a, more details can be found in Tables S1 and S2.

Both RhoA and Wnt-5a mRNA and protein expression were positively associated with International Federation of Gynecology and Obstetrics (FIGO) stage (III/IV vs. I/II; Figure 1E,L, $p<0.05$ ) and degree of differentiation in ovarian carcinoma (poorly differentiated carcinomas $v s$. other subtypes; Figure 1F,M, $p<0.05$ ). However, no correlations were observed between RhoA or Wnt-5a expression and pathological subtype in ovarian carcinoma (Figure $1 \mathrm{G}, \mathrm{N}, p>0.05$ ). RhoA mRNA expression was significantly positively correlated with Wnt-5a mRNA expression in ovarian carcinoma (Figure $1 \mathrm{H}$, $\left.p=0.001, R^{2}=0.1669\right)$.

\subsection{Effects of RhoA or Wnt-5a Knockdown on Phenotype and Expression of Wnt-5a Signaling-Related} Molecules in Ovarian Carcinoma Cells

Figure 2A shows that RhoA mRNA and protein expression was highest in OVCAR3 cells; therefore, we used OVCAR3 cells for RhoA small interfering RNA (siRNA) transfection. RhoA and Wnt-5a mRNA and protein expression in OVCAR3 RhoA or Wnt-5a siRNA transfectants were significantly reduced as compared to negative control (NC) and mock-transfected cells (Figure 2B,C, $p<0.05$ ), as demonstrated by real-time PCR and western blotting. Both RhoA and Wnt-5a siRNA transfectants exhibited significantly slower growth than NC and mock-transfected cells (Figure 2D, $p<0.05$ ), based on the CCK-8 assay. Propidium iodide (PI) staining and flow cytometry revealed significant induction of $\mathrm{G}_{1}$ arrest in RhoA and Wnt-5a siRNA transfectants (Figure 2E, G, $p<0.05$ ). Knockdown of either RhoA or Wnt-5a induced significantly higher levels of apoptosis (Figure $2 \mathrm{~F}, \mathrm{H}$, $p<0.05$ ), indicated by annexin V-fluorescein isothiocyanate (FITC) staining; suppressed lamellipodia formation (Figure 3A), indicated by loss of F-actin structure in cells stained with Alexa Fluor ${ }^{\circledR}$ (Invitrogen, Carlsbad, CA, USA) 568 phalloidin; and reduced cell migration in the wound healing assay (Figure 3B, $p<0.05$ ) and invasion in the Transwell invasion assay (Figure 3C, $p<0.05$ ) in comparison with NC and mock-transfected cells. In cells transfected with RhoA siRNA, there was reduced expression of Akt, Bcl-xL, vascular endothelial growth factor (VEGF), p70 S6 kinase (p70S6k), and survivin mRNA in OVCAR3 cells (Figure 4A,B, $p<0.05$ ); similar effects were observed in cells transfected with Wnt-5a siRNA. Western blotting showed that knockdown of either RhoA or Wnt-5a also downregulated the levels of Akt, PI3K, Bcl-xL, VEGF, p70S6k and survivin protein expression (Figure 4C). 
Figure 1. Correlation of RhoA and Wnt-5a mRNA and protein expression with the pathogenesis and aggressiveness of ovarian carcinoma. (A-G,I-M) RhoA and Wnt-5a mRNA and protein expression quantified in normal fallopian tube epithelium (NFTE), benign tumors $(\mathrm{Be})$, primary ovarian carcinomas $(\mathrm{Ca})$, and metastatic omentum $(\mathrm{Om})$ using real-time PCR (A,B) and western blotting (I); Significantly higher RhoA and Wnt-5a mRNA and protein expression found in ovarian carcinomas compared to benign tumors or normal fallopian tube epithelium $(\mathbf{C}, \mathbf{J})$ and in metastatic omentum foci than in ovarian carcinomas (D,K); Positive association between RhoA and Wnt-5a mRNA and protein expression levels with FIGO staging (E,L, stage I/II vs. stage III/IV) and degree of differentiation (F,M, poorly differentiated (Por) vs. moderately differentiated (Mod) and well differentiated (Well)) in ovarian carcinoma; $(\mathbf{G}, \mathbf{N})$ No correlations between RhoA or Wnt-5a mRNA and protein expression and pathological subtype in ovarian carcinoma; and (H) Significant positive correlation between RhoA mRNA expression and Wnt-5a mRNA expression in ovarian carcinoma $\left(R^{2}=0.1669\right)$. $* p<0.05$.

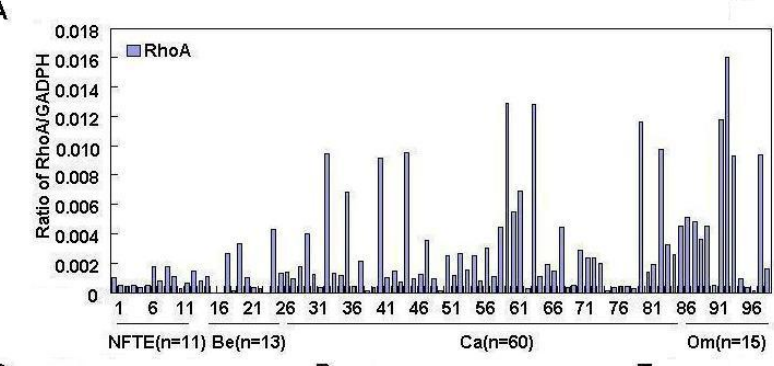

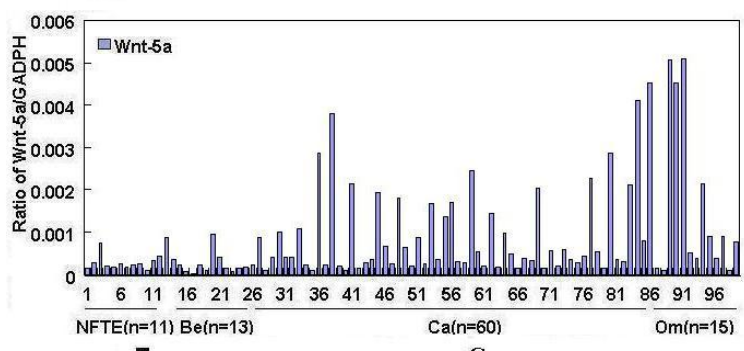
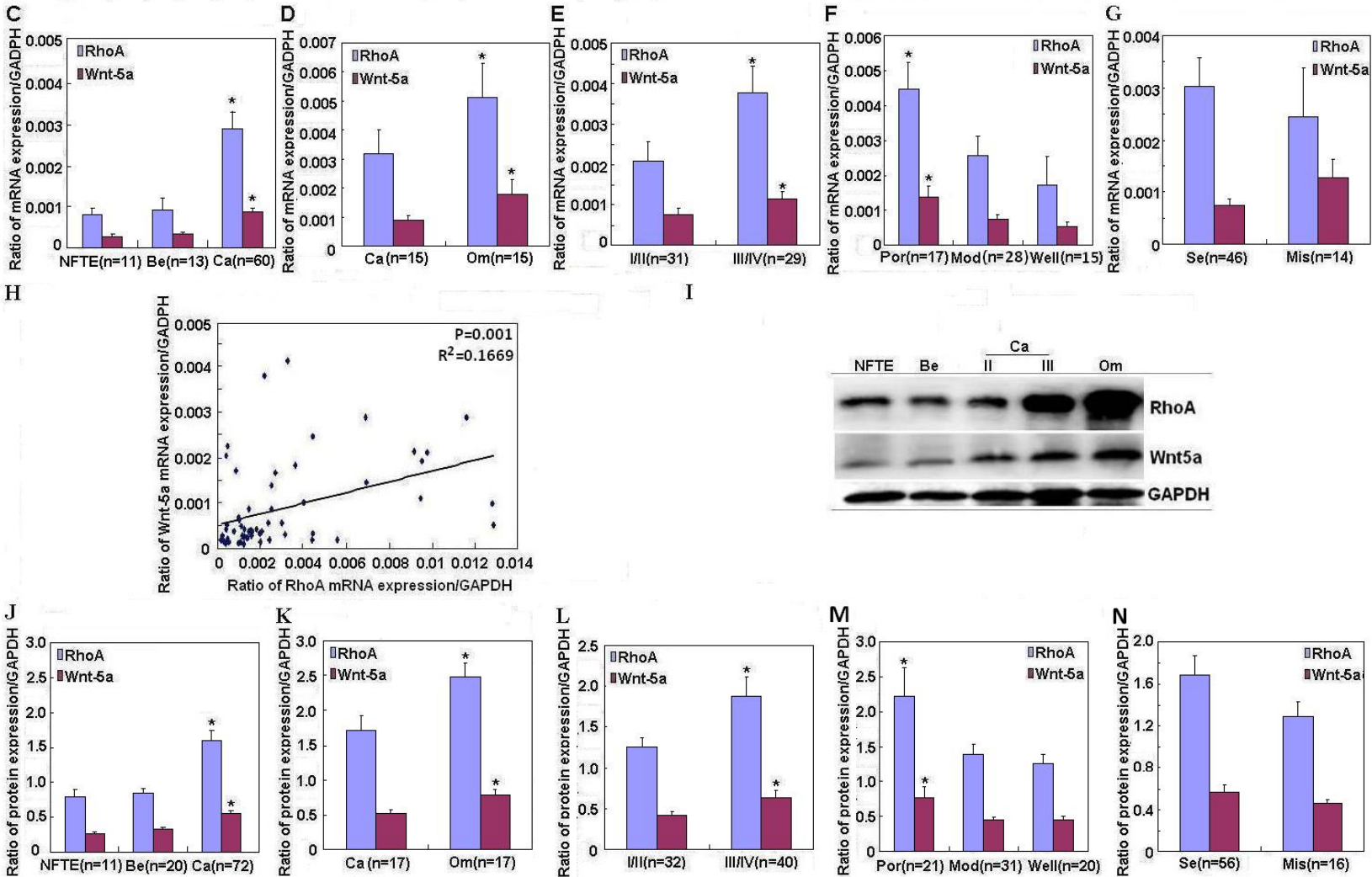
Figure 2. Effects of RhoA or Wnt-5a knockdown on mRNA and protein expression, cell proliferation, cell cycle, and apoptosis of ovarian carcinoma cells. (A) OVCAR3 cells were used for RhoA siRNA transfection because RhoA mRNA and protein expression was highest in this cell line; RhoA siRNA (B) or Wnt-5a siRNA transfection (C) significantly reduced RhoA and Wnt-5a expression in OVCAR3 cells compared to negative control (NC) or mock-transfected cells. OVCAR3 cells transfected with RhoA siRNA or Wnt-5a siRNA exhibited significantly reduced cell viability (D) in comparison with NC and mock-transfected cells; (E,G) Significantly increased $G_{1}$ arrest in OVCAR3 cells transfected with RhoA siRNA or Wnt-5a siRNA in comparison with NC and mock-transfected cells; and $(\mathbf{F}, \mathbf{H})$ Significantly increased apoptosis in OVCAR3 cells transfected with RhoA siRNA or Wnt-5a siRNA in comparison with NC and mock-transfected cells. ${ }^{*} p<0.05$. Results are representative of three separate experiments; data are expressed as the mean \pm standard deviation.
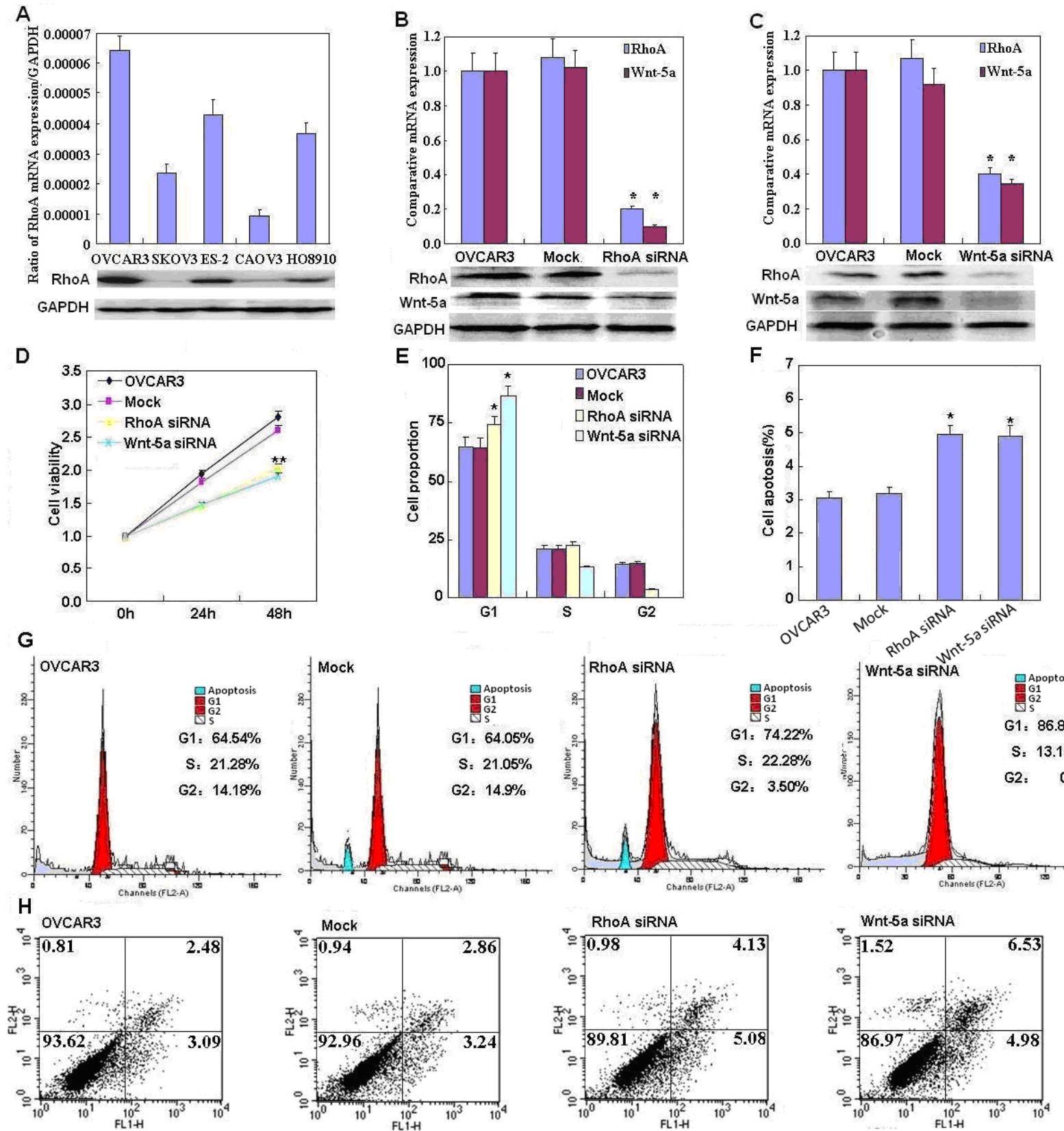
Figure 3. Effects of RhoA or Wnt-5a knockdown on the invasive ability of OVCAR3 cells. (A-C) OVCAR3 cells transfected with RhoA siRNA or Wnt-5a siRNA exhibited suppressed lamellipodia formation (A) magnification $\times 40$; significantly reduced migration $(\mathbf{B})$, Scale bar $=200 \mu \mathrm{m}$; and significantly reduced cell invasion $(\mathbf{C})$ in comparison with $\mathrm{NC}$ and mock-transfected cells, magnification $\times 20 .{ }^{*} p<0.05$. Results are representative of three separate experiments; data are expressed as the mean \pm standard deviation.
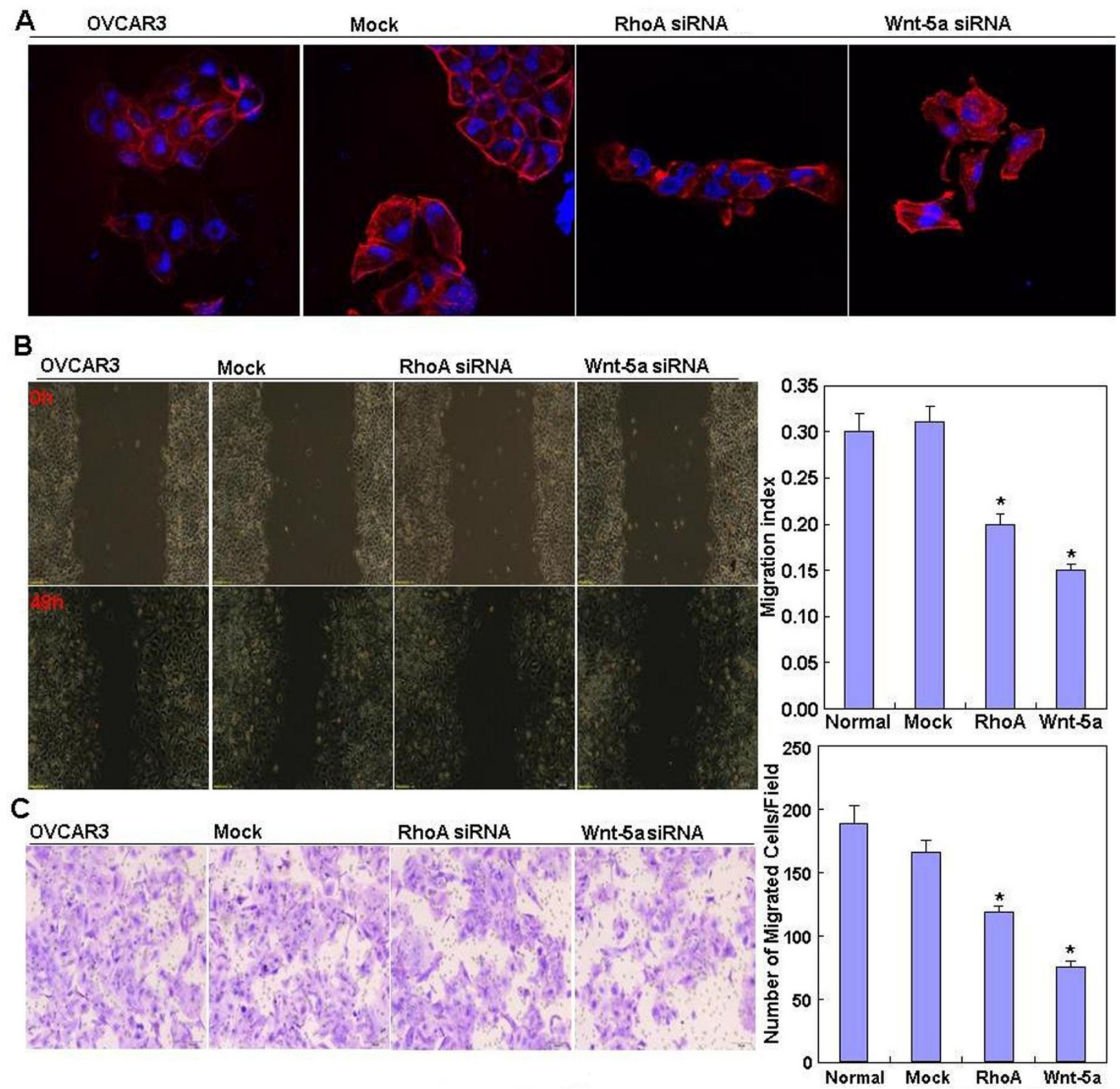
Figure 4. OVCAR3 cell mRNA and protein expression profiles after RhoA or Wnt-5a knockdown. (A-C) OVCAR3 cells transfected with RhoA siRNA or Wnt-5a siRNA expressed significantly lower levels of Akt, Bcl-xL, VEGF, p70S6k, and survivin mRNA $(\mathbf{A}$ and $\mathbf{B})$ and protein $(\mathbf{C})$ in comparison with $\mathrm{NC}$ and mock-transfected cells. ${ }^{*} p<0.05$.

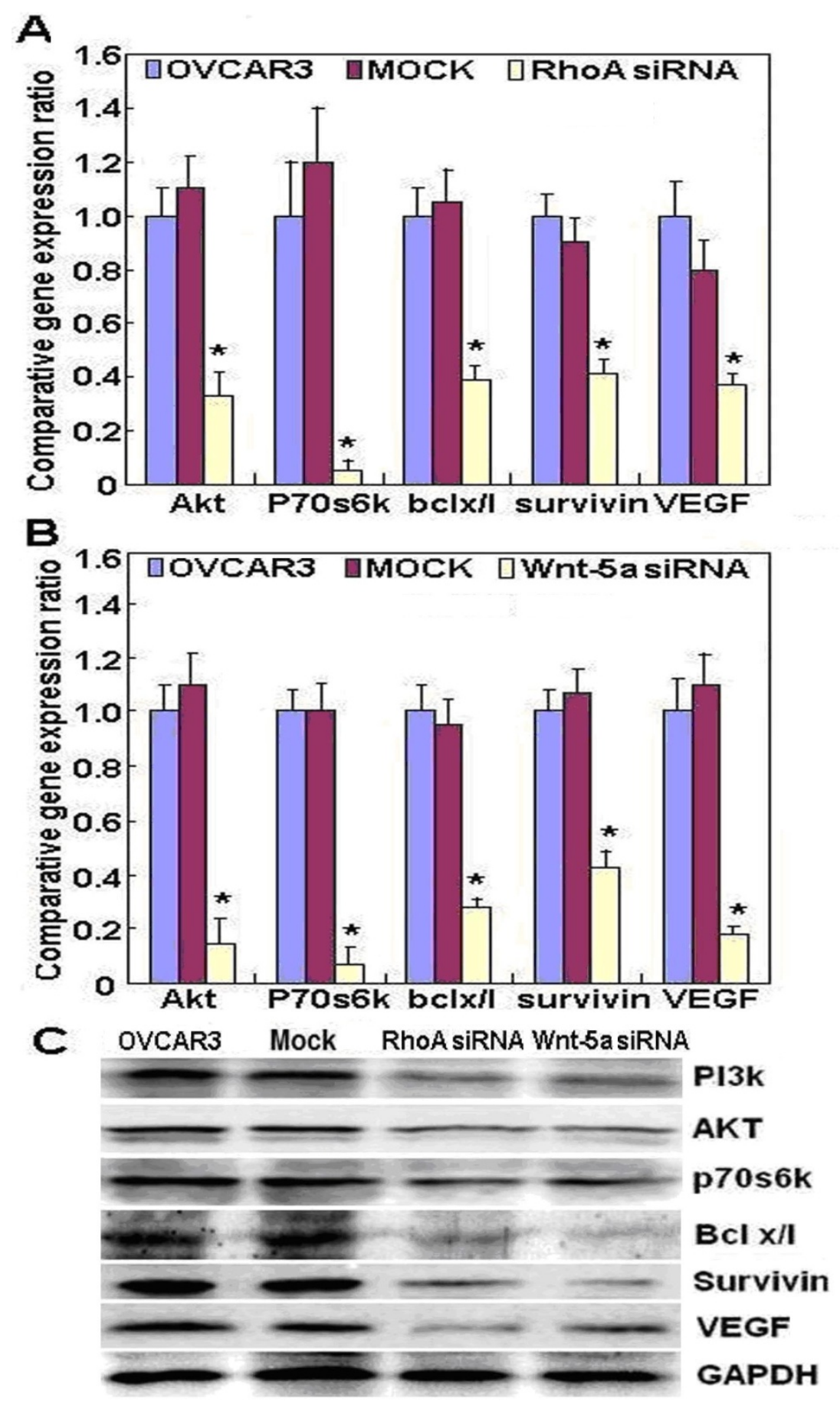

\section{Discussion}

As reviewed by Hall [4], RhoA exerts multiple functions in tumor metastasis by orchestrating the action of multiple downstream effectors and promoting the degradation and reconstruction of the extracellular matrix. Ellenbroek et al. [10] reported that RhoA protein was overexpressed in head and neck cancers and gastric cancer, indicating that RhoA upregulation is closely associated with 
carcinogenesis. Our study showed that RhoA expression was significantly upregulated in ovarian carcinomas compared to normal fallopian tube epithelium and benign tumors, in accordance with Horiuchi et al. [11]. However, RhoA expression was upregulated in metastatic omentum compared to ovarian carcinomas; additionally, RhoA expression was positively associated with FIGO stage and degree of differentiation in ovarian carcinoma. These findings indicate that $R h o A$ overexpression might affect ovarian carcinogenesis and subsequent progression.

In addition, we found that RhoA knockdown induced $\mathrm{G}_{1}$ arrest and apoptosis, decreased lamellipodia formation, and reduced cell migration and invasion, which indicates that RhoA downregulation may suppress the aggressive phenotypes of ovarian carcinoma cells. Our data are in agreement with that of Peacock et al. [12], who reported that RhoA promotes reorganization of the actin cytoskeleton, regulates cell shape and attachment, and coordinates cell motility and actomyosin contractility. We also found that knockdown of RhoA significantly reduced both mRNA and protein expression of Akt, p70S6k, Bcl-xL, survivin, and VEGF in OVCAR3 cells. According to the literature, these molecules are involved in the regulation of proliferation, apoptosis, invasion, and metastasis of cancer cells [13-15]. Taken together, these results suggest that RhoA may promote proliferation, anti-apoptosis, and invasion by modulating the expression of these genes.

$W n t-5 a$ is a prototypic ligand that activates a $\beta$-catenin-independent pathway in Wnt signaling. Wnt-5a has been demonstrated to exert differential effects on cancer development. It promotes cancer progression and metastasis in malignant melanoma, breast cancer, and gastric cancer [8,16-18]. Kurayoshi et al. [18] reported that Wnt-5a stimulated cell motility, invasiveness, and aggressiveness in some cancer cells, suggesting that it exerts oncogenic effects. Li et al. [19] also reported that Wnt-5a exerted stem cell functions in lung cancer. In contrast, some reports indicate that in view of its ability to inhibit cell proliferation, motility, or invasion in thyroid tumor and colorectal cancer cell lines, $W n t-5 a$ acts as a tumor suppressor [20-24]. Our study showed that Wnt-5a was also significantly upregulated in ovarian carcinomas compared to normal fallopian tube epithelium and benign tumors and in metastatic omentum compared to primary carcinomas, in line with Peng et al. [25] and Badiglian et al. [26], indicating that Wnt-5a may be involved in the tumorigenesis and progression of ovarian cancer. The functional experiments also suggested that Wnt-5a knockdown attenuated the aggressiveness of ovarian carcinoma, including proliferation, anti-apoptosis, migration, and invasion, by modulating phenotype-related molecules such as Akt, PI3K, Bcl-xL,VEGF, p70S6k, and survivin.

Although Strutt et al. [27] reported that Wnt-mediated cell migration appeared to require RhoA activation, and Qian et al. [28] found that Wnt-5a activated planar cell polarity (PCP) through a RhoA-dependent process, leading to control of cellular movement, we found that Wnt-5a knockdown significantly reduced both RhoA mRNA and protein expression. RhoA mRNA expression was positively correlated with that of Wnt-5a mRNA in ovarian carcinoma tissue. These results suggest that there may be a positive correlation between RhoA and Wnt-5a expression in ovarian carcinoma. However, further study of validation in in vitro models may better explain the obtained results.

In conclusion, we demonstrated for the first time that RhoA and Wnt-5a may be associated with ovarian carcinogenesis and apoptosis inhibition; there might be positive correlation between RhoA and Wnt-5a expression. RhoA has potential as a biomarker of tumorigenesis, differentiation, and progression in ovarian carcinoma. 


\section{Patients and Methods}

\subsection{Cell Culture and Transfection}

The ovarian carcinoma cell lines CAOV3 (serous adenocarcinoma), OVCAR3 (serous cystic adenocarcinoma), SKOV3 (serous papillary cystic adenocarcinoma), HO8910 (serous cystic adenocarcinoma), and ES-2 (clear cell carcinoma) were purchased from ATCC (Manassas, VA, USA). They were maintained in RPMI 1640 (ES-2, HO8910, OVCAR3), DMEM (CAOV3), or McCoy's 5A (SKOV3) media supplemented with 10\% fetal bovine serum (FBS), 100 units $/ \mathrm{mL}$ penicillin, and $100 \mu \mathrm{g} / \mathrm{mL}$ streptomycin in a humidified atmosphere of $5 \% \mathrm{CO}_{2}$ at $37^{\circ} \mathrm{C}$. OVCAR3 cells were transfected with RhoA siRNA or Wnt-5a siRNA (Sigma-Aldrich, St. Louis, MO, USA). The RhoA target sequences were 5'-GUUUAUUAAUCUUAGUGUAdTdT-3' (sense) and 5'-UACACUAAGAUUAAUAAACdTdT-3' (antisense). The Wnt-5a target sequences were 5'-CUGACUACUGCGUGCGCAAdTdT-3' (sense) and 5'-UUGCGCACGCAGUAGUCAGdTdT-3' (antisense). The NC siRNA sequences were 5'-UUCUCCGAACGUGUCACGUTT-3' (sense) and 5'-ACGUGACACGUUCGGAGAATT-3' (antisense). Transfected cells were collected by centrifugation, rinsed with phosphate-buffered saline (PBS), and total proteins were extracted by sonication in radioimmunoprecipitation assay (RIPA) buffer.

\subsection{Cell Viability Assay}

Cell Counting Kit-8 (CCK-8; Dojindo, Tokyo, Japan) was used to determine the numbers of viable cells. Briefly, $2.0 \times 10^{3}$ cells/well were seeded into 96-well plates and allowed to adhere. The next day, the media was changed to starvation medium (total volume $100 \mu \mathrm{L} /$ well) for an additional $24 \mathrm{~h}$, and cells were challenged with $200 \mathrm{ng} / \mathrm{mL}$ RhoA/Wnt-5a/Mock siRNAs (R \& D Systems, Minneapolis, $\mathrm{MN}$, USA). At the appropriate time points, $10 \mu \mathrm{L}$ CCK-8 solution was added to each well, the plates incubated for $4 \mathrm{~h}$, and the absorbance values measured at $450 \mathrm{~nm}$ using a microplate reader.

\subsection{Cell Cycle Analysis}

After incubation for $48 \mathrm{~h}$ at $37{ }^{\circ} \mathrm{C}$ in an atmosphere of $5 \% \mathrm{CO}_{2}$, cells were trypsinized, harvested, washed twice in PBS, and fixed in $10 \mathrm{~mL}$ ice-cold ethanol for at least $2 \mathrm{~h}$. Cells were then washed twice in PBS and incubated with $500 \mu \mathrm{L}$ RNase $(0.25 \mathrm{mg} / \mathrm{mL})$ at $37{ }^{\circ} \mathrm{C}$ for $30 \mathrm{~min}$. Cells were pelleted by centrifugation, resuspended in $50 \mu \mathrm{g} / \mathrm{mL}$ Propidium Iodide (PI, KeyGen, Nanjing, China), and incubated at $4{ }^{\circ} \mathrm{C}$ in the dark for $30 \mathrm{~min}$. The PI signal was examined using flow cytometry.

\subsection{Flow Cytometric Apoptosis Assay}

Flow cytometry was performed using cells stained with PI and FITC-labeled annexin V (KeyGen, Nanjing, China) to detect phosphatidylserine externalization as an endpoint indicator of early apoptosis, as described in the manufacturer's instructions. Briefly, after incubation for $48 \mathrm{~h}$ at $37{ }^{\circ} \mathrm{C}$ in an atmosphere of $5 \% \mathrm{CO}_{2}$, cells were washed twice with cold PBS, resuspended in $1 \times$ binding buffer at $1 \times 10^{6}$ cells $/ \mathrm{mL}$, and incubated with $200 \mu \mathrm{L}$ Binding Buffer and $10 \mu \mathrm{L}$ Annexin V-FITC. Samples were gently vortexed, incubated for $15 \mathrm{~min}$ at $25^{\circ} \mathrm{C}$ in the dark, $300 \mu \mathrm{L}$ Binding Buffer and $5 \mu \mathrm{L}$ PI were added to each tube, and flow cytometry was performed within $1 \mathrm{~h}$. 


\subsection{Wound Healing Assay}

Cells were seeded at $1.0 \times 10^{6}$ cells/well in 6-well culture plates. After cells had grown to confluence, the confluent monolayer in each well was scratched with a pipette tip, washed three times with PBS, and cultured in FBS-free medium. Cells were photographed at 0, 12, 24, $48 \mathrm{~h}(n=9)$; the scratch area was measured using Image $J$ software (National Institutes of Health, Bethesda, MD, USA). The wound healing rate = (Area of original wound - Area of actual wound at different times)/Area of original wound $\times 100 \%$.

\subsection{Cell Invasion Assay}

A thin layer of Matrigel ( $40 \mu \mathrm{L}$ from $8 \mathrm{mg} / \mathrm{mL}$ stock solution; Becton-Dickinson Labware, Bedford, MA, USA) was overlaid on the upper surface of $6.5-\mathrm{mm}$ Transwell chambers $(8-\mu \mathrm{m}$ pore size; BD Bioscience, San Jose, CA, USA). The Matrigel was allowed to solidify by incubating the plates for $4 \mathrm{~h}$ at $37^{\circ} \mathrm{C}$. Culture medium was added to the bottom Transwell chamber. Cells were resuspended in serum-free RPMI 1640 (HyClone, Logan, UT, USA) at $2.5 \times 10^{5}$ cells $/ \mathrm{mL}$, and $5 \times 10^{4}$ cells were added to the top Transwell chambers. Following 48-h incubation, cells that had not invaded through the Matrigel were removed from the upper surface using cotton swabs. Cells that had invaded through the Matrigel and reached the bottom surface of the filters were fixed in methanol and stained with $0.1 \%$ crystal violet. Invasion was quantified by counting the number of cells under a Olympus fluorescence microscope (Tokyo, Japan) equipped with a 16-square reticle. The surface area of this grid was $1 \mathrm{~mm}^{2}$. Five separate fields were counted for each filter $(n=3)$; the total numbers of cells were compared among experimental groups using the $t$-test with the assumption of two-tail distribution and two samples with equal variance. A difference of $p<0.05$ was considered statistically significant.

\subsection{Immunofluorescent Staining}

Cells were grown on glass coverslips, fixed with PBS containing 4\% formaldehyde for $10 \mathrm{~min}$, permeabilized with $0.2 \%$ Triton X-100 in PBS for 10 min at room temperature, washed with PBS, and incubated with Alexa Fluor ${ }^{\circledR} 568$ phalloidin (Invitrogen, Carlsbad, CA, USA) overnight at $4{ }^{\circ} \mathrm{C}$ to visualize the lamellipodia. Nuclei were stained with $1 \mu \mathrm{g} / \mathrm{mL}$ diamidino phenylindole (Sigma-Aldrich, St. Louis, MO, USA) for $15 \mathrm{~min}$ at $37{ }^{\circ} \mathrm{C}$. Coverslips were mounted using SlowFade ${ }^{\circledR}$ Gold antifade reagent (Invitrogen, Carlsbad, CA, USA) and cells were observed using a laser confocal microscope (Olympus, Tokyo, Japan).

\subsection{Ovarian Epithelium and Carcinoma Samples}

Between August 2003 and December 2011, normal fallopian tube epithelium, ovarian epithelial benign tumors (serous and mucinous cystadenoma), primary carcinoma specimens, and metastatic omentum were obtained from patients undergoing surgical resection at the Department of Gynecology, The First Affiliated Hospital of China Medical University. The tumor specimens were microscopically confirmed by pathologists. The average age at surgery was 51.6 years (range 20-81 years). Each ovarian carcinoma specimen was evaluated according to the International Federation of Gynecology and Obstetrics (FIGO) staging system (2009). The histological architecture of ovarian 
carcinoma was defined in terms of World Health Organization classification. Samples were frozen immediately in liquid nitrogen and stored at $-80{ }^{\circ} \mathrm{C}$ until analysis. None of the patients underwent chemotherapy, radiotherapy, or adjuvant treatment before surgery. Informed consent was obtained from all subjects; the China Medical University Ethics Committee approved the study.

\subsection{Real-Time Reverse Transcriptase PCR}

Total RNA was extracted from ovarian carcinoma cell lines and ovarian tissues using TRIzol (Takara, Shiga, Japan) according to the manufacturer's protocol. Total RNA ( $2 \mu \mathrm{g}$ ) was reverse transcribed to complementary DNA (cDNA) using avian myeloblastosis virus transcriptase and random primers (Takara, Shiga, Japan). The oligonucleotide primers for PCR were based on GenBank sequences (Table S3). Real-time PCR amplification of the cDNA was performed in $20-\mu \mathrm{L}$ reactions according to the SYBR Premix Ex Taq ${ }^{\mathrm{TM}}$ II kit (Takara, Shiga, Japan); glyceraldehyde-3-phosphate dehydrogenase $(G A P D H)$ was used as the internal control.

\subsection{Western Blotting}

Protein was extracted in ice-cold RIPA lysis buffer and its concentration was determined by protein assay kit (Bio-Rad Laboratories, Hercules, CA, USA). Denatured proteins (100 $\mu \mathrm{g})$ were separated on sodium dodecyl sulfate-polyacrylamide gels, transferred to Hybond membranes (Amersham, Munich, Germany), and blocked overnight in 5\% skimmed milk in Tris-buffered saline with Tween 20 (TBST). For immunoblotting, membranes were incubated for $15 \mathrm{~min}$ with antibodies against RhoA (Abcam, Cambridge, UK), Wnt-5a, PI3K, Akt, Bcl-xL, survivin, VEGF (Santa Cruz Biotechnology, Santa Cruz, CA, USA), or p70s6k (T421/s424; Cell Signaling Technology, Danvers, MA, USA). Then, the membranes were rinsed with TBST and incubated with anti-mouse, anti-rabbit, or anti-goat IgG antibodies conjugated to horseradish peroxidase (1:1000; Dako, Carpinteria, CA, USA) for $15 \mathrm{~min}$. All incubations were performed in a microwave oven (Oriental Rotor, Tokyo, Japan) with intermittent irradiation. Bands were visualized on X-ray film (Fujifilm, Tokyo, Japan) using ImageQuant LAS 4000 (Fujifilm, Tokyo, Japan) and ECL Plus detection reagents (Santa Cruz Biotechnology, Santa Cruz, CA, USA). Subsequently, the membranes were washed with WB Stripping Solution ( $\mathrm{pH}$ 2-3; Nacalai Tesque, Tokyo, Japan) for $1 \mathrm{~h}$ and reprobed using mouse anti-GAPDH antibody (Sigma-Aldrich, St. Louis, MO, USA) as the internal control.

\subsection{Statistical Analysis}

Statistical analysis was performed using the Spearman correlation test for ranked data and the Mann-Whitney $U$ test and paired samples $t$-test to compare the means of different groups. $p<0.05$ were considered statistically significant. SPSS 10.0 software (SPSS, Chicago, IL, USA) was used to analyze all data.

\section{Conclusions}

Our findings indicate that RhoA and Wnt-5a are associated with ovarian carcinogenesis and apoptosis inhibition; there might be a positive correlation between RhoA and Wnt-5a expression. RhoA is a potential tumorigenesis, differentiation, and progression biomarker in ovarian carcinoma. 


\section{Acknowledgments}

This study was supported by Shenyang Outstanding Talent Foundation of China; Shenyang Science and Technology Grant (F11-264-1-10; F12-277-1-01); the Project Supported by Scientific Research Fund of Liaoning Provincial Education Department (L2010633); Liaoning Science and Technology Grant (2009225008-11; 2013021077); and the Natural Scientific Foundation of China (81172371; 81202049).

\section{Conflicts of Interest}

The authors declare no conflict of interest.

\section{References}

1. Piek, J.M.; Kenemans, P.; Zweemer, R.P.; van Diest, P.J.; Verheijen, R.H. Ovarian carcinogenesis, an alternative theory. Gynecol. Oncol. 2007, 107, 355.

2. Piek, J.M.; van Diest, P.J.; Verheijen, R.H. Ovarian carcinogenesis: An alternative hypothesis. Adv. Exp. Med. Biol. 2008, 622, 79-87.

3. Yu, L.-L.; Gu, J.-Y. Advances in the role of Rho sub-family in tumor invasion. Fudan Univ. J. Med. Sci. 2010, 37, 617-619.

4. Hall, A.; Rho, G.T. Pases and the actin cytoskeleton. Science 1998, 279, 509-514.

5. Der Mardirossian, C.; Bokoch, G.M. GDIs: Central regulatory molecules in Rho GTPase activation. Trends Cell Biol. 2005, 15, 356-363.

6. Olofsson, B. Rho guanine dissociation inhibitors: Pivotal molecules in cellular signalling. Cell. Signal. 1999, 11, 545-554.

7. Liu, J.; Zhang, Y.; Xu, R.; Du, J.; Hu, Z.; Yang, L.; Chen, Y.; Zhu, Y.; Gu, L. PI3K/Akt-dependent phosphorylation of GSK3$\beta$ and activation of RhoA regulate Wnt5a-induced gastric cancer cell migration. Cell. Signal. 2013, 25, 447-456.

8. Zhu, Y.; Tian, Y.; Du, J.; Hu, Z.; Yang, L.; Liu, J.; Gu, L. Dv12-dependent activation of Daam1 and RhoA regulates Wnt5a-induced breast cancer cell migration. PLoS One 2012, 7, e37823.

9. Kikuchi, A.; Yamamoto, H.; Sato, A.; Matsumoto, S. Wnt5a: Its signalling, functions and implication in diseases. Acta Physiol. (Oxf.) 2012, 204, 17-33.

10. Ellenbroek, S.I.; Collard, J.G. Rho GTPases: Functions and association with cancer. Clin. Exp. Metastasis 2007, 24, 657-672.

11. Horiuchi, A.; Imai, T.; Wang, C.; Ohira, S.; Feng, Y.; Nikaido, T.; Konishi, I. Up-regulation of small GTPases, RhoA and RhoC, is associated with tumor progression in ovarian carcinoma. Lab. Investig. 2003, 83, 861-870.

12. Peacock, J.G.; Miller, A.L.; Bradley, W.D.; Rodriguez, O.C.; Webb, D.J.; Koleske, A.J. The Abl-related gene tyrosine kinase acts through p190RhoGAP to inhibit actomyosin contractility and regulate focal adhesion dynamics upon adhesion to fibronectin. Mol. Biol. Cell 2007, 18, 3860-3872.

13. Merajver, S.D.; Usmani, S.Z. Multifaceted role of Rho proteins in angiogenesis. J. Mammary Gland Biol. Neoplasia 2005, 10, 291-298. 
14. Ip, C.K.M.; Cheung, A.N.Y.; Ngan, H.Y.S.; Wong, A.S.T. p70 S6 kinase in the control of actin cytoskeleton dynamics and directed migration of ovarian cancer cells. Oncogene 2011, 30, 2420-2432.

15. Denoyelle, C.; Albanese, P.; Uzan, G.; Hong, L.; Vannier, J.P.; Soria, J.; Soria, C. Molecular mechanism of the anti-cancer activity of cerivastatin, an inhibitor of HMG-CoA reductase, on aggressive human breast cancer cells. Cell Signal. 2003, 15, 327-338.

16. Weeraratna, A.T.; Jiang, Y.; Hostetter, G.; Rosenblatt, K.; Duray, P.; Bittner, M.; Trent, J.M. Wnt5a signaling directly affects cell motility and invasion of metastatic melanoma. Cancer Cell 2002, 1, 279-288.

17. Bittner, M.; Meltzer, P.; Chen, Y.; Jiang, Y.; Seftor, E.; Hendrix, M.; Radmacher, M.; Simon, R.; Yakhini, Z.; Ben-Dor, A.; et al. Molecular classification of cutaneous malignant melanoma by gene expression profiling. Nature 2000, 406, 536-540.

18. Kurayoshi, M.; Oue, N.; Yamamoto, H.; Kishida, M.; Inoue, A.; Asahara, T.; Yasui, W.; Kikuchi, A. Expression of Wnt-5a is correlated with aggressiveness of gastric cancer by stimulating cell migration and invasion. Cancer Res. 2006, 66, 10439-10448.

19. Li, X.; Jia, Y.; Zhang, W.; Zhang, Y.; Li, B.; Huang, M; Bao, F.; Wu, J.; Lou, Y. The Research progress about Wnt pathway on lung cancer stem cells. Chin. J. Lung Cancer 2011, 14, 695-698.

20. Dejmek, J.; Dejmek, A.; Safholm, A.; Sjolander, A.; Andresson, T. Wnt-5a protein expression in primary dukes B colon cancers identifies a subgroup of patients with good prognosis. Cancer Res. 2005, 65, 9142-9146.

21. Kremenevskaja, N.; von Wasielewski, R.; Rao, A.S.; Schöfl, C.; Andersson, T.; Brabant, G. Wnt-5a has tumor suppressor activity in thyroid carcinoma. Oncogene 2005, 24, 2144-2154.

22. Veeman, M.T.; Axelrod, J.D.; Moon, R.T. A second canon: Functions and mechanisms of $\beta$-catenin-independent Wnt signaling. Dev. Cell 2003, 5, 367-377.

23. Kurayoshi, M.; Yamamoto, H.; Izumi, S.; Kikuchi, A. Post-translational palmitoylation and glycosylation of Wnt-5a are necessary for its signaling. Biochem. J. 2007, 402, 515-523.

24. Kikuchi, A.; Yamamoto, H. Tumor formation due to abnormalities in the $\beta$-catenin-independent pathway of Wnt signaling. Cancer Sci. 2008, 99, 202-208.

25. Peng, C.; Zhang, X.; Yu, H.; Wu, D.; Zheng, J. Wnt5a as a predictor in poor clinical outcome of patients and a mediator in chemoresistance of ovarian cancer. Int. J. Gynecol. Cancer 2011, 21, 280-288.

26. Badiglian, F.L.; Oshima, C.T.; de Oliveira Lima, F.; de Oliveira Costa, H.; de Sousa Damião, R.; Gomes, T.S.; Gonçalves, W.J. Canonical and noncanonical Wnt pathway: A comparison among normal ovary, benign ovarian tumor and ovarian cancer. Oncol. Rep. 2009, 21, 313-320.

27. Strutt, D.; Weber, U.; Mlodzik, M. The role of RhoA in tissue polarity and Frizzled signalling. Nature 1997, 387, 292-295.

28. Qian, D.; Jones, C.; Rzadzinska, A.; Mark, S.; Zhang, X.; Steel, K.P.; Dai, X.; Chen, P. Wnt5a functions in planar cell polarity regulation in mice. Dev. Biol. 2007, 306, 121-133.

(C) 2013 by the authors; licensee MDPI, Basel, Switzerland. This article is an open access article distributed under the terms and conditions of the Creative Commons Attribution license (http://creativecommons.org/licenses/by/3.0/). 